\title{
EDITORIAL
}

\section{Perinatal mental health around the world: a new thematic series ${ }^{\dagger}$}

\author{
John Cox
}

Emeritus Professor of Psychiatry, Keele University, UK. Email: john6.cox@gmail.com

Conflicts of interest: None

\section{${ }^{+}$See this issue.}

Keywords. Perinatal psychiatry; perinatal; international; postnatal depression.

First received 6 Nov 2019 Accepted 6 Nov 2019

\section{doi:10.1192/bji.2019.32}

(c) The Author 2019. This is an Open Access article, distributed under the terms of the Creative Commons Attribution licence Commons Attibution licence (http://creativecommons.org/ licenses/by/4.0/), which permits unrestricted re-use, distribution, and reproduction in any medium provided the original work is properly cited.
International multiprofessional teams in primary and secondary care have much to teach psychiatrists, researchers and service planners about the perinatal mental health field. This editorial introduces a new series of papers in BJPsych International on perinatal mental health around the world.

Politicians, in spite of - or because of - Brexit turmoil have recently held prolonged debates in the House of Commons on perinatal mental illness and early years family support, ${ }^{1,2}$ when they spoke from personal experience and demanded improved services. Perinatal mental health advocacy by international organisations such as the Marcé Society for Perinatal Mental Health and the Global Alliance for Maternal Mental Health, is not only establishing an implementation research base but is also enabling a more universal as well as culture-specific understanding of service and research priorities, as papers in this new thematic series 'Perinatal mental health around the world' will illustrate.

\section{A spotlight on three countries}

The papers from India, ${ }^{3}$ Sweden $^{4}$ and Italy ${ }^{5}$ published in this issue provide readers with insights as to why at national and local level the plight and mortality of new parents with mental disorders are so frequently unnoticed. They point out that the human costs to society of this treatment gap are considerable. They suggest caution, however, before assuming that one size will fit all, and illustrate the different professional skill mixes in primary and secondary care across national boundaries - as well as the impact of family support on the need for residential parent and baby units. Curiously, the effect of perinatal mental illness on fathering is only beginning to be fully acknowledged - and research on grandparenting is in its infancy.

Each paper has emphasised the need to develop comprehensive national guidelines and pathways to care across the primary/secondary care boundary - and regard this as a current priority. The absence of access to specialist perinatal psychiatric services is striking.

\section{India}

In India, Ganjekar and colleagues ${ }^{3}$ have drawn attention to the absence of mental health in research priorities for maternal, newborn and child health and nutrition by national funding bodies. They nevertheless underline the truism that pioneer developments such as the mother and baby unit in Bangalore initiated by Prabha Chandra, and the new service developments outlined by her colleagues in Kerala, demonstrate models of care that can move mountains. Their detailed perinatal research priorities are pertinent not just for Indian funding agencies but also the wider international community.

\section{Sweden}

Birgitta Wickberg in Gothenburg, and her colleagues from Uppsala and Stockholm, ${ }^{4}$ describe in their paper from Sweden how they have already pioneered universal primary care screening and a therapy service which incorporates the Edinburgh Postnatal Depression Scale (EPDS) and offers of 'listening visits' by trained midwives, nurses and psychologists. They also ask: where are the psychiatrists and where is the tertiary care? Strikingly they remind us that Sweden is now one of the most multiracial countries in Europe and highlight the need for new skills in this transcultural field, which is so politically sensitive.

\section{Italy}

Pietro Grussu in Padua, and his colleagues in Rome and Venice, ${ }^{5}$ remind us of the frequency of the non-psychotic mental disorders of childbirth and draw attention to the present drivers of change in Italy, which include public concern about the number of mothers who kill themselves during pregnancy or in the puerperium, as well as greater awareness of violence by partners or family members. The lack of specialist perinatal facilities is implicit in this paper.

\section{A call for thinking outside of the box}

Taken together, it is hoped that these multiprofessional papers from Europe and India will provoke the reader to think outside the box of narrow specialism and to maintain an integrative focus for clinical work. At the heart of perinatal mental health around the world there are family relationships, fathers as well as mothers - and an infant growing as a person with a name.

The Editor of this Journal will welcome submissions from other countries which will inform multiprofessional teams in primary and secondary 
care - and which may also implicitly or explicitly redefine and clarify the role of psychiatrists in this burgeoning field.

\section{References}

1 Williams P. Perinatal mental illness. Hansard, HC Deb, 2018, 645, $19 \mathrm{Jul}$

2 Leadsom A. Early years family support. Hansard, HC Deb, 2019, 663,16 Jul.
3 Ganjekar S, Thekkethayyil AV, Chandra PS (2019) Perinatal mental health around the world: priorities for research and service development in India. BJPsych International, this issue.

4 Wickberg B, Bendix M, Wetterholm MB, et al (2019) Perinatal mental health around the world: priorities for research and service development in Sweden. BJPsych International, this issue.

5 Grussu et al (2019) Perinatal mental health around the world: priorities for research and service development in Italy. BJPsych International, this issue.

\title{
THEMATIC \\ Perinatal mental health around the PAPER world: priorities for research and service development in India
}

\author{
Sundarnag Ganjekar, ${ }^{1}$ Anilkumar Viswananthan Thekkethayyil ${ }^{2}$ and \\ Prabha S. Chandra ${ }^{3}$ (i)
}

\begin{abstract}
Associate Professor, Department of Psychiatry, National Institute of Mental Health and Neurosciences, Bangalore, India 2Professor, Department of Psychiatry, Government Medical College, Thiruvananthapuram, India

${ }^{3}$ Professor, Department of Psychiatry, National Institute of Mental Health and

Neurosciences, Bangalore, India. Email: chandra@nimhans.acin

Conflicts of interest: None.

Keywords. Perinatal psychiatry; India; LAMIC; postpartum depression; health systems.

First received 14 May 2019 Final revision 19 Jul 201 Accepted 10 Oct 2019

doi:10.1192/bji.2019.26

(c) The Authors 2019. This is an Open Access article, distributed under the terms of the Creative Commons Attribution licence (http://creativecommons.org/ licenses/by/4.0/), which permits unrestricted re-use, distribution, and reproduction in any medium, provided the original work is properly cited.
\end{abstract}

A video abstract is available for this article at: vimeo.com/ bjpsych/bji-2019-26

\begin{abstract}
Maternal mental health disorders are a significant problem for mother-infant dyads in India, but have not received the attention that they should. However, recent major developments hold promise: the increase in coverage of the District Mental Health Programme; the growing emphasis in public health systems on newborn health; integration of maternal mental health into the Reproductive and Child Health Programme in the state of Kerala; and the Mental Health Care Act 2017, which mandates mother-infant joint care when a mother is admitted for mental illness, will lead to policy changes in services. Innovative implementation and translational research is needed to generate knowledge to strengthen maternal mental healthcare systems and improve maternal and child outcomes. Valuable 'research rupees' should be spent on ensuring equity of resources for physical and mental healthcare of mothers and providing optimal environments for every mother-infant dyad.
\end{abstract}

The priorities of India's Reproductive and Child Health (RCH) Programme have, thus far, been to reduce maternal and infant mortality and improve newborn health. A comprehensive public health programme for perinatal mental health is yet to evolve and there are very few dedicated perinatal psychiatry services. In recent years, several obstetricians, public health experts and psychiatrists have been advocating for prioritising perinatal mental health. The 2018 Biennial Scientific Meeting of the International Marcé
Society in Bangalore was one such attempt, which helped put perinatal mental health in the spotlight.

Two major developments herald well for the future of perinatal mental health in India. First is the increasing coverage of the District Mental Health Programme, which will be able to provide an effective referral and training platform, and second, the growing emphasis in public health systems on newborn health. The government's National Health Mission has also started discussing ways of integrating perinatal mental health into existing mother and child programmes.

\section{What should our model for perinatal mental health services look like?}

India is a large country, and, healthcare being under the control of individual states, there are wide variations in the provision of both mental healthcare and maternal healthcare. Therefore, it might be too ambitious to have a single plan that works countrywide. Bagadia \& Chandra $^{1}$ have proposed a stepped-care approach and the need to develop adequate care pathways. However, to do that, healthcare professionals first need to be sensitised to the importance and relevance of perinatal mental health. They should then be able to identify pregnant and postpartum women with mental health problems and also those who are at risk. The first step will therefore be to enhance the level of awareness of perinatal mental health among health professionals. Simultaneously, developing or adapting simple tools for detection will be a priority. Complex screening tools developed in the West often do not translate easily into Indian languages, have rating scales that are difficult for low-literacy 